
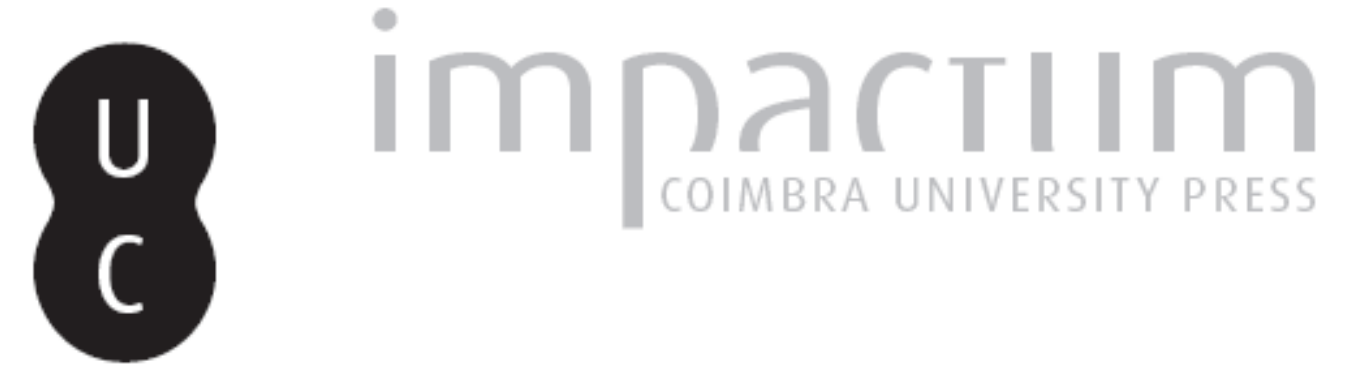

O dano sexual: contributo para a avaliação médico-legal dos danos na pessoa

Autor(es): $\quad$ Santos, L.; Magalhães, T.; Costa, D. Pinto da; Matos, E.

Publicado por: Imprensa da Universidade de Coimbra

URL persistente:

URI:http://hdl.handle.net/10316.2/33078

DOI:

DOI:http://dx.doi.org/10.14195/1647-8630_18_2

Accessed : $\quad$ 26-Apr-2023 09:42:02

A navegação consulta e descarregamento dos títulos inseridos nas Bibliotecas Digitais UC Digitalis, UC Pombalina e UC Impactum, pressupõem a aceitação plena e sem reservas dos Termos e Condições de Uso destas Bibliotecas Digitais, disponíveis em https://digitalis.uc.pt/pt-pt/termos.

Conforme exposto nos referidos Termos e Condições de Uso, o descarregamento de títulos de acesso restrito requer uma licença válida de autorização devendo o utilizador aceder ao(s) documento(s) a partir de um endereço de IP da instituição detentora da supramencionada licença.

Ao utilizador é apenas permitido o descarregamento para uso pessoal, pelo que o emprego do(s) título(s) descarregado(s) para outro fim, designadamente comercial, carece de autorização do respetivo autor ou editor da obra.

Na medida em que todas as obras da UC Digitalis se encontram protegidas pelo Código do Direito de Autor e Direitos Conexos e demais legislação aplicável, toda a cópia, parcial ou total, deste documento, nos casos em que é legalmente admitida, deverá conter ou fazer-se acompanhar por este aviso.

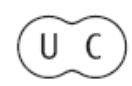




\title{
0 dano sexual. Contributo para a avaliação médico-legal dos danos na pessoa
}

\author{
L. Santos ${ }^{1,2}$, T. Magalhães ${ }^{1,3}$, D. Pinto da Costa ${ }^{1,2}$, E. Matos ${ }^{3}$
}

\section{Introdução}

A sexualidade humana constitui uma área essencial das nossas vidas, não só como uma via para a reprodução, como também na perspectiva da afectividade e da gratificação pessoal. Assim, disfunções de carácter sexual, qualquer que seja a sua etiologia, podem ter enormes repercussões do ponto de vista individual (como a diminuição da auto-estima ou a depressão) e em termos familiares, podendo culminar na separação de um casal.

Muitos destes problemas têm soluções terapêuticas, mas a falta de informação da comunidade em geral sobre o assunto, bem como os tabus sociais ainda existentes, levam a que tanto doentes como terapeutas se inibam, muitas vezes, não o abordando.

A avaliação das perturbações do desempenho sexual, nomeadamente das disfunções sexuais, impõe muitas dificuldades, mas muito se tem investigado nos últimos anos para que se possa não só, compreender a etiologia e os mecanismos psico-fisiológicos subjacentes a cada uma delas, como também adequar o tratamento a cada caso particular. A evolução da medicina sexual relaciona-se não só com a necessidade de todos compreendermos uma área ainda não totalmente explorada, mas também com a assunção de que os problemas da intimidade de cada um podem ter soluções que passam por um diagnóstico preciso e atempado.

Um grande número destas situações relacionam-se com traumatismos, como é o caso dos acidentes de viação. Ora, quando nestes casos de etiologia traumática se apura eventual responsabilidade de terceiros, poderá haver lugar à avaliação médico-legal do dano corporal resultante para estas

\footnotetext{
Instituto Nacional de Medicina Legal, I.P. - Delegação do Norte

Faculdade de Medicina da Universidade do Porto

3 Instituto de Ciências Biomédicas Abel Salazar - Universidade do Porto
} 
vítimas, sendo em sede de Direito Civil que a maioria destas situações são avaliadas, tendo como objectivo a reparação integral dos danos sofridos, tal como consignado no artigo $562^{\circ}$ do Código Civil Português. O momento da realização do exame médico-legal deve ser, por vários motivos, considerado privilegiado para o diagnóstico desta sequela.

O desconhecimento, a vergonha, o receio do ridículo, impedem que as questões da sexualidade sejam, por norma, abordadas. Tal não devia acontecer, uma vez que a saúde sexual se inclui na noção geral de saúde e, como tal, não deveria nunca ser negligenciada. Mas a medicina legal poderá constituir uma última hipótese para a abordagem médica desta problemática, pelo que os peritos médico-legais devem ter sempre presente a possibilidade da existência de um DS e ter a noção da importância de uma correcta avaliação de todas as sequelas do indivíduo, particularmente aquelas que ao serem normalmente esquecidas em avaliações anteriores, podem afectar a vítima para sempre. No entanto, a avaliação do DS nem sempre se realiza e quando tem lugar, nem sempre obedece a critérios rigorosos, pelo que importa analisar estes aspectos com mais pormenor.

\section{a) 0 Dano Sexual}

No que respeita aos danos na esfera da sexualidade, está prevista, no âmbito do Direito Civil, a valoração do Dano Sexual (DS), enquanto parâmetro de dano independente, não económico ou extra-patrimonial. Mas, em termos médico-legais, a avaliação deste dano constitui um grande desafio pericial; primeiro, porque a sua objectivação nem sempre é possível, o que se traduz, por vezes, em critérios díspares em termos de avaliação; segundo, porque o tema em avaliação pertence à esfera íntima da vítima, a ele se associando certos preconceitos, pelo que muitas vezes o mesmo nem chega sequer a ser abordado na avaliação médico-legal; finalmente, porque passa por vezes despercebido ou é subvalorizado, face à gravidade objectiva de outras sequelas.

É, no entanto, indiscutível que para um ressarcimento total dos danos na pessoa é essencial avaliar a existência deste eventual dano. É também claro, que tal avaliação deve ser sempre feita segundo os mesmos critérios periciais, de modo a que se garanta não só rigor na avaliação, como justiça na decisão final quanto à reparação a atribuir.

A avaliação do DS está, em grande parte, relacionada com a capacidade de diagnosticar uma disfunção sexual. As dificuldades neste diagnóstico, vastamente referidas na literatura de urologia, ginecologia e psiquiatria são, em grande medida, aquelas com que se depara a Medicina Legal. Nos últimos 20 anos, muitos esforços têm sido feitos, na tentativa de colmatar estas adversidades (4). Assim, importa salientar que, se por um lado, a medicina legal 
não é a única especialidade com dificuldades na avaliação do DS, por outro, a consciencialização global dessas limitações está claramente a dinamizar o estudo destas disfunções, criando instrumentos que poderão permitir colmatar tais dificuldades, estimulando também a participação neste esforço colectivo de tomada de consciência para um problema ainda demasiado oculto.

\section{b) Disfunções sexuais}

A disfunção sexual constitui uma situação em que a função sexual está afectada por factores biológicos, psicológicos e sociais. Um distúrbio numa ou mais destas áreas pode resultar em disfunção sexual (12).

A presença de uma disfunção sexual cria no indivíduo que a sofre ansiedade, medo e vergonha, sentimentos que, por sua vez, condicionam o agravamento e a perpetuação desta disfunção $(1,12,14)$.

Durante várias décadas, os dois sistemas de classificação de disfunções sexuais mais utilizados foram a Classificação Internacional das Doenças (actualmente CID-10) e o Manual Diagnóstico e Terapêutico das Doenças Mentais (actualmente DSM-IV-TR) adoptados, respectivamente, pela Organização Mundial de Saúde (OMS) e pela American Psychiatric Association's (4).

As quatro categorias major de disfunções sexuais em ambos os sistemas acima referidos, são desordens a nível do desejo, excitação, orgasmo e dor $(4,13)$.

O DSM-IV-TR é, ainda hoje, o sistema de classificação mais utilizado (12). De acordo com os seus critérios, são consideradas sete categorias major de disfunções sexuais (2):

a. perturbações do desejo sexual (desejo sexual hipoactivo e aversão sexual);

b. perturbações da excitação sexual (perturbação da excitação sexual na mulher e disfunção eréctil no homem);

c. perturbações do orgasmo (perturbação do orgasmo na mulher, perturbação do orgasmo no homem e ejaculação precoce);

d. perturbações de dor sexual (dispareunia e vaginismo);

e. perturbações sexuais secundárias a um estado físico geral;

f. disfunções sexuais induzidas por substâncias;

g. disfunções sexuais sem outra especificação.

Como já referido, as duas últimas décadas foram profícuas em encontros científicos e investigações levadas a cabo na área da sexualidade humana. Na sequência destes vários encontros, actualmente são já seis os sistemas de classificação mais utilizados em medicina sexual. Além do CID-10 e do DSM-IV-TR, estão ainda disponíveis as classificações do National Institute of Health Consensus Conference on Impotence, da American Foundation for Urologic Diseases, do International Consensus Conference on Women's Sexual 
Dysfunction e do First and Second International Consultations on Sexual Dysfunctions. (4).

Estas classificações são, genericamente, semelhantes entre si. É importante referir que a principal evolução está relacionada com a inclusão da percepção da disfunção pelo indivíduo, ou seja, no diagnóstico de uma disfunção sexual passou a ser considerado importante não apenas a limitação apresentada pelo indivíduo, mas, acima de tudo, a forma como o indivíduo entende e vivencia a sua limitação $(5,11)$. Em consonância com isto, surgiram novas ferramentas de avaliação, nomeadamente questionários auto-aplicados. Estes questionários são cada vez mais utilizados no diagnóstico das disfunções sexuais (5). Salienta-se, assim, a valorização da subjectividade, demonstrando que a evolução não vai tanto no sentido da total objectivação das patologias mas, antes, no sentido de uma visão personalizada sobre as mesmas. A ciência caminha, claramente, para o estudo dos doentes em detrimento do estudo das doenças.

\section{c) Disfunções sexuais pós-traumáticas}

A vivência de um evento traumático tem consequências por vezes devastadoras na vida do indivíduo. As consequências do trauma constituem um grande desafio, uma vez que as situações traumáticas são, actualmente, uma das causas mais importantes de morbilidade física e psico-social crónica. Nesta matéria, a medicina legal assume um papel importante pela experiência e reflexão que resultam do trabalho diário com vítimas de trauma (9). Nestes casos, a sexualidade é também muitas vezes afectada, cabendo ao perito avaliar a presença de uma perturbação do desempenho sexual e a sua relação com o trauma sofrido.

A disfunção sexual pós-traumática não é uma designação utilizada com frequência, no entanto, em muitos estudos, e mesmo na literatura médica clássica, encontram-se referências a disfunções sexuais que, pela sua etiologia, cabem nesta designação.

De acordo com os critérios do DSM-IV-TR, algumas das disfunções sexuais pós-traumáticas poderiam ser incluídas nas disfunções de tipo adquirido, secundário a factores psicológicos ou secundário a factores combinados. Dentro deste último subgrupo, é importante salientar que as especificações relativas a disfunções sexuais secundárias a um estado físico geral contemplam muitas situações onde é possível incluir casos de disfunções pós-traumáticas, designadamente: lesões do lobo temporal; epilepsia do lobo temporal; patologia traumática da medula espinal; neuropatia periférica; parésia generalizada; procedimentos cirúrgicos, nomeadamente resseção cólica abdomino-perineal, simpatectomia, cirurgia aorto-ilíaca e cistectomia radical $(2,12)$. Acresce que alguns casos de disfunções sexuais induzidas por substâncias (2), poderão ser 
consideradas pós-traumáticas, caso a terapêutica subjacente à disfunção esteja associada ao tratamento de uma patologia decorrente do evento traumático.

As disfunções podem, pois, resultar apenas do trauma psicológico, apenas do traumatismo orgânico, ou de ambos.

\section{d) Aspectos médico-legais do Dano Sexual}

O DS constitui um dano não económico (ou extra-patrimonial), avaliado e reparado em sede de Direito Civil, e corresponde à limitação total ou parcial do nível de desempenho/gratificação de natureza sexual, decorrente das sequelas físicas e/ou psíquicas, não se incluindo aqui os aspectos relacionados com a capacidade de procriação (15).

É quantificado numa escala com sete graus de gravidade crescente (1/7 a 7/7), cuja fundamentação é obrigatória não só no capítulo da "Discussão" mas, também, através da descrição correcta e pormenorizada das queixas (funcionais e situacionais) e sequelas, nos respectivos capítulos (15).

O DS pode manifestar-se através de perturbação do desejo, da excitação, do orgasmo ou de dor relacionada directamente com o acto sexual ou provocada por este, devido a sequelas resultantes do evento traumático ou, ainda, por dificuldades de posicionamento, devido também às sequelas traumáticas.

Significa isto que, tendo sempre em conta o estado anterior da vítima, o papel do perito inclui a avaliação de eventual disfunção sexual pós-traumática ou perturbação da actividade sexual na dependência de sequelas consequentes ao evento traumático. Ou seja, avaliar o nexo de causalidade entre a perturbação do desempenho sexual descrita e o evento traumático em estudo.

Pode considerar-se que o conceito de DS é um conceito mais alargado do que o conceito genérico de disfunção sexual, uma vez que, em algumas situações se encontram alterações da actividade sexual do indivíduo não contempladas nas várias classificações de disfunções sexuais.

\section{Objectivos}

O objectivo do presente trabalho é, em termos gerais, analisar os critérios de valoração do DS numa população de peritos portugueses, identificando os pontos fracos da mesma, tendo em vista contribuir para o repensar da metodologia de avaliação do DS.

Os objectivos específicos são os seguintes:

1. Identificar os tipos de lesões associadas aos casos que apresentam perturbações do desempenho sexual, numa população de traumatizados por acidente de viação; 
2. Avaliar a qualidade da valoração deste dano pelos peritos, tendo em conta a sua descrição e os critérios científicos usados para a sua fundamentação/justificação;

3. Avaliar a forma como os tribunais repararam este dano;

4. Estabelecer a correlação entre os danos valorados pelo perito e pelo tribunal.

\section{Material e Métodos}

Realizou-se um estudo retrospectivo a partir dos relatórios periciais elaborados no âmbito do Direito Civil, nos serviços médico-legais (Delegação e Gabinetes) do norte de Portugal, de Janeiro de 2002 a Dezembro de 2005 $(\mathrm{n}=156)$; analisaram-se, ainda, as respectivas sentenças judiciais $(\mathrm{n}=47)$. Consideraram-se os seguintes critérios de inclusão: (a) exames no âmbito de Direito Civil; (b) consequentes a acidente de viação; (c) com relatórios periciais concluídos, (d) nos quais era feita referência a queixas do foro da sexualidade e/ou atribuído DS.

Para a realização da base de dados foi utilizado o programa informático Excel 2003 e para o tratamento estatístico dos dados foi usado o SPSS 15.0 para Windows.

\section{Resultados}

A população estudada era maioritariamente masculina (58.3\%), com idade média de 36 anos (Mín=14, Máx=68, DP=13.6), sem antecedentes patológicos de relevo (96.2\%).

As lesões resultantes distribuíram-se pelos seguintes grupos (Gráfico 1):

Grupo I: traumatismos de crânio e/ou pescoço, excepto aqueles em que está associado traumatismo raquimedular $(n=58 ; 36 \%)$;

Grupo II: traumatismo raquimedular isoladamente ou associado a outras lesões $(\mathrm{n}=39 ; 25 \%)$ - atingimento neurológico em $41 \%(\mathrm{n}=16)$;

Grupo III: traumatismo dos membros isolado ou associado a outros traumatismos, exceptuando os do crânio e os raquimedulares $(\mathrm{n}=54 ; 34.6 \%)$;

Grupo IV: traumatismos do tórax e abdómen e traumatismos da face $(\mathrm{n}=5 ; 3.2 \%)$; destes, 65 casos tiveram traumatismo crânioencefálico (42\%), 29 lesões da bacia e/ou anca (18.6\%) e 7 lesões dos órgãos genitais e/ou vias urinárias (4.4\%). 


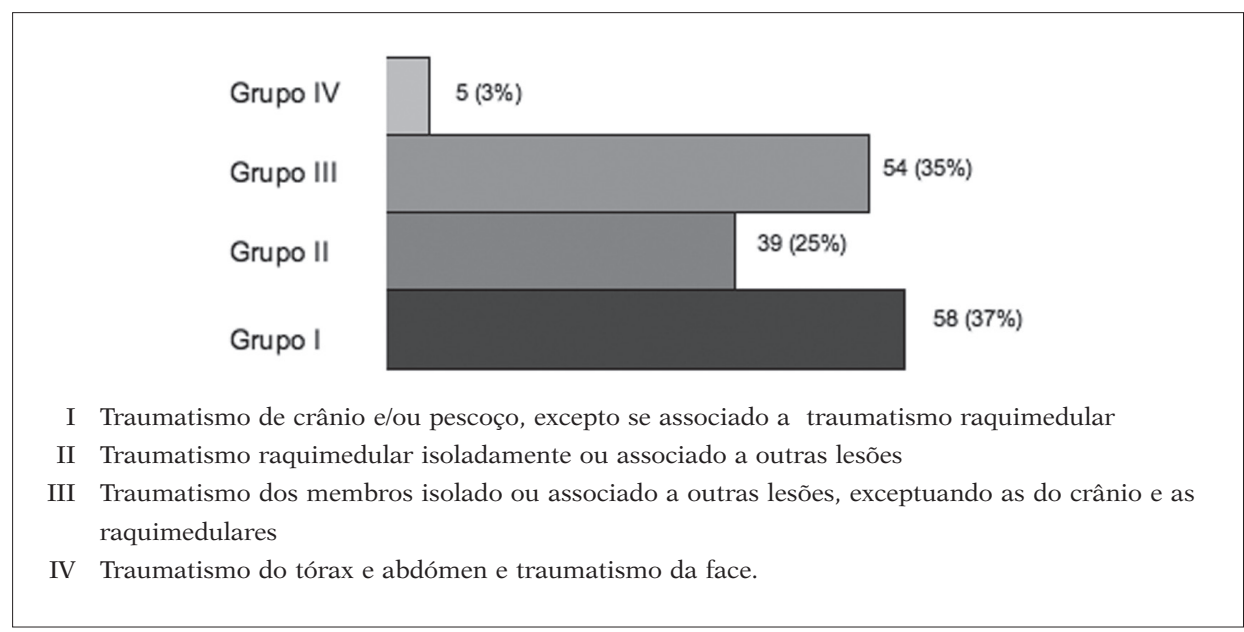

Gráfico 1. Distribuição das lesões segundo os grupos definidos (n=156)

Dos 156 casos em que havia referência a eventual disfunção sexual, o DS foi atribuído em apenas 111 (71\%), em 3 deles de forma qualitativa e nos restantes valorado quantitativamente.

Na totalidade dos casos em que o DS foi atribuído ( $n=111)$, este não foi descrito a nível das queixas em $19.8 \%$ dos casos. Nos restantes $80.2 \%(n=89)$, e na maior parte das vezes, foi feita referência a dificuldades durante o acto sexual, devido a queixas álgicas residuais, na sequência das várias lesões sofridas (29.2\%) (Tabela 1).

Em 27 dos casos em que houve atribuição de DS (24.3\%), este não foi fundamentado ou justificado pelo perito. Destes, o DS foi contudo descrito a nível das queixas em 21 casos (77.8\%) (Tabela 2).

Verificou-se ainda, que nos casos em que foi feita a fundamentação do DS ( $n=84,76 \%$ ) (Tabela 3 ), a mesma nem sempre encontrou suporte a nível da sua descrição no capítulo das queixas (n=16; 19.1\%) (Tabela 2).

\begin{tabular}{lcc}
\hline \multicolumn{1}{c}{ Queixas do desempenho sexual } & n & \% \\
\hline Queixas álgicas não genitais & 26 & 29.2 \\
Perturbações da erecção & 21 & 23.6 \\
Perturbações do desejo & 10 & 11.2 \\
Perturbações do desejo e queixas álgicas não genitais & 10 & 11.2 \\
Outras queixas & 22 & 25.1 \\
\hline
\end{tabular}

Tabela 1. Descrição pelos peritos das queixas do desempenho sexual (n=89) 


\begin{tabular}{lccc}
\hline \multicolumn{1}{c}{ DS (\%) } & Justificado & N/ Justificado & Totais \\
Descrito & 61 & 19 & 80 \\
N/ Descrito & 15 & 5 & 20 \\
\hline Totais & 76 & 24 & 100 \\
\hline
\end{tabular}

Tabela 2. Critérios de valoração do DS - Fundamentação da avaliação (n=111)

\begin{tabular}{lcc}
\hline \multicolumn{1}{c}{ Justificação do DS } & n & $\%$ \\
\hline Queixas álgicas não genitais & 21 & 25 \\
Perturbações do desejo & 10 & 12 \\
Perturbações da erecção & 8 & 9.5 \\
Perturbações do desejo e queixas álgicas não genitais & 3 & 3.5 \\
Outras justificações & 42 & 50 \\
\hline
\end{tabular}

Tabela 3. Justificação pelos peritos do DS atribuído ( $n=84)$

Para a melhor fundamentação deste dano, os peritos apoiaram-se, muito excepcionalmente $(n=6)$, em exames de outras especialidades, designadamente: (a) Urologia ( $\mathrm{n}=1)$; (b) Psiquiatria $(\mathrm{n}=3)$; (c) Urologia e Psiquiatria $(\mathrm{n}=2)$.

Analisando a amostra global $(\mathrm{n}=156)$, constatou-se que foi atribuído Quantum Doloris (QD) em todos os casos, Incapacidade Permanente Geral (IPG) em 155 (99.4\%), Dano Estético (DE) em 119 (76.3\%) e Prejuizo de Afirmação Pessoal (PAP) em 61 (39.1\%) (Tabela 4).

\begin{tabular}{lccc}
\hline \multicolumn{1}{c}{ Parâmetros de dano } & $\begin{array}{c}\text { Atribuído } \\
(\mathrm{n} ; \%)\end{array}$ & Mín. & Máx. \\
\hline Incapacidade Permanente Geral & $155 ; 99.4$ & 5 & 100 \\
Dano Futuro & $22 ; 14.1$ & 5 & 15 \\
Quantum Doloris & $156 ; 100$ & 1 & 7 \\
Dano Estético & $119 ; 76.3$ & 1 & 7 \\
Prejuízo de Afirmação Pessoal & $61 ; 39.1$ & 1 & 5 \\
\hline
\end{tabular}

Tabela 4. Outros parâmetros de dano valorados $(n=156)$

Relativamente às decisões judiciais ( $\mathrm{n}=47$ ), o DS foi considerado como um parâmetro de dano relevante para a definição da indemnização em apenas 1 caso e, em apenas 9 havia referências a queixas relacionadas com o foro sexual.

\section{Discussão}

A sexualidade faz parte integrante da natureza humana e daí a sua importância, que vai para além das necessidades reprodutivas. Acresce a este facto, a circunstância de que os humanos associam o sexo ao amor e aos afectos, 
razão pela qual o sexo e a sexualidade mantêm a sua importância mesmo com o avançar da idade (5), assumindo um papel preponderante na manutenção harmoniosa de relações interpessoais e familiares, e no bem-estar individual.

Apesar deste facto, a eventual existência de DS não parece ser analisada por rotina nas perícias médico-legais, como sugerem os resultados deste estudo, em que há referência à presença de perturbações do desempenho sexual apenas em 4\% dos casos. A este propósito, registe-se como exemplo curioso, que num exaustivo trabalho, efectuado em área geográfica aproximada e pelos mesmos serviços médico-legais, que analisa os diferentes parâmetros de dano em relação aos acidentes de viação em geral (7), o DS não é considerado, o que pode corroborar a ideia de que este, efectivamente, não é suficientemente tido em conta.

As razões serão várias, e já foram sendo apontadas. O ensino actual da medicina em Portugal não inclui, na maioria dos estabelecimentos de ensino, formação capaz na área da medicina sexual, disto resultando um natural desconhecimento destas questões, o que acarretará uma desvalorização da sexualidade na avaliação geral dos doentes.

Por outro lado, a avaliação de uma perturbação do desempenho sexual é uma tarefa complexa, que exige conhecimento e experiência. Mas tal não justifica a sua não avaliação, uma vez que, constitui obrigação do perito médico-legal procurar adquirir capacidades, nas diversas áreas necessárias ao desempenho da sua função (8).

Nos exames realizados em sede de Direito Civil, cabe ao perito avaliar todas as sequelas decorrentes do evento traumático, conhecendo a doutrina médico-legal e a existência de vários danos a avaliar; um deles é, sem dúvida, o DS $(6,10,15)$, apesar da Tabela de Indemnizações portuguesa ${ }^{4}$ parecer ignorá-lo.

Não será exigível, que todos os peritos sejam capazes de avaliar sozinhos todas as situações em que existe uma perturbação do desempenho sexual, nem tal seria desejável, porque em muitos casos importa esclarecer o diagnóstico através do recurso a outras especialidades e a exames complementares de diagnóstico, mas é fundamental que os peritos sejam capazes de diagnosticar todas as possíveis disfunções sexuais e, sempre que necessário, orientem as vítimas para os adequados exames de especialidade (Neurologia, Endocrinologia, Ginecologia, Urologia/Andrologia, Psiquiatria/Sexologia e/ou Psicologia, entre outros) e/ou complementares. Esta é aliás, como acima referido, uma obrigação do perito, já que, face ao desenvolvimento actual da medicina, não é possível ter em simultâneo conhecimentos aprofundados das matérias relativas a todas as especialidades médicas, deve por isso, o perito médico, apoiar-se sempre que adequado, em pareceres de outras especialidades (8).

4 Portaria n. ${ }^{\circ}$ 377/2008 de 26 de Maio 
A determinação do nexo de causalidade no caso do DS é um momento que encerra grandes dificuldades. Efectivamente, a determinação deste nexo é particularmente complexa, na maioria dos casos, dada a difícil objectivação do dano e comprovação do estado anterior, bem como as múltiplas etiologias das eventuais disfunções sexuais pós-traumáticas.

No entanto, a impossibilidade de objectivação não pode justificar, em todos os casos, a não atribuição do dano, uma vez que um trauma físico e/ ou psíquico pode condicionar queixas persistentes, que tanto o exame clínico, como eventuais exames complementares, não consigam diagnosticar (em alguns casos justificado pelo estado actual da nossa tecnologia). Nestes casos, o médico não encontra para as queixas um substrato objectivo, mas pode dizer se estas queixas são ou não cientificamente explicáveis, ou seja, se são plausíveis (6), devendo admitir, se o forem, o nexo de causalidade.

Relativamente à reparação do DS, a análise realizada não deixa dúvidas de que são muito raras as vezes em que as questões relativas à intimidade sexual são abordadas pelos advogados e juízes. Várias podem ser as explicações para este facto.

Se, como já referido, os próprios profissionais de saúde têm algumas dificuldades em abordar estas questões, nomeadamente por falta de formação, não será surpreendente que para os advogados e juízes estes assuntos sejam ainda de mais difícil abordagem.

Como refere Foucault (3), a lei está habituada a lidar com a sexualidade no sentido punitivo, havendo uma longa tradição e evolução neste sentido, nomeadamente, em relação aos crimes sexuais. Na avaliação do DS, o que se pede ao Direito é que lembre sempre a sexualidade, não na sua vertente punitiva, mas antes como parte inerente e essencial para o bem-estar do indivíduo, tão importante que da sua normalidade está dependente a manutenção da espécie humana.

Pode parecer que ultrapassa as competências da Medicina Legal alertar a comunidade jurídica para estas questões, no entanto, tal objectivo será mais fácil de atingir do que poderá aparentar. A realização sistemática de uma análise séria deste dano, descrevendo-o, valorando-o e justificando-o de forma adequada, alertará estes profissionais para a sua existência.

A mudança não será imediata, mas o nosso objectivo maior não é a rapidez, mas sim a eficácia, e essa por vezes demora o seu tempo. Acreditamos que poderá valer a pena. 


\section{Conclusões}

O carácter íntimo da matéria em avaliação, a dificuldade de objectivação do dano e, por vezes, a ausência de abordagem destas situações, conduzem a uma subavaliação do DS. No entanto, este tipo de dano pode ter grande relevância para a qualidade de vida dos doentes, pelo que não pode deixar de ser diagnosticado, tratado e reparado.

A avaliação do DS pressupõe a existência de atitudes e conhecimentos relativamente à abordagem das questões do foro da sexualidade que, com base no estudo efectuado, parecem carecer de um maior desenvolvimento, pelo menos no grupo de peritos sobre o qual foi efectuada a análise. Efectivamente, os resultados sugerem que não são seguidos quaisquer critérios na avaliação deste dano, ficando por vezes mesmo esquecida aquela norma fundamental à avaliação pericial de qualquer dano, a fundamentação ou justificação da valoração feita.

A circunstância do relatório pericial não contribuir, em quase nada, para a produção da sentença no que ao DS diz respeito, pode estar relacionada com o facto da avaliação deste dano, nos relatórios periciais, não parecer obedecer, como se referiu, a critérios rigorosos, sendo que num relevante número de casos a sua valoração é feita sem uma qualquer fundamentação (24\%), mas resultará, sobretudo, do facto das vítimas e seus advogados não estarem sensibilizadas nem atentos à possibilidade deste dano poder ser reparado, pelo que o mesmo não aparece geralmente quesitado.

As dificuldades de avaliação deste dano, demonstradas no presente estudo, são reveladoras da necessidade de um maior trabalho de definição de normas procedimentais quanto aos seus critérios de avaliação, bem como uma melhor formação e sensibilização dos peritos sobre a importância desta matéria. De facto, os resultados do presente estudo permitiram concluir que:

1. As perturbações a nível da sexualidade são referidas em 4\% dos relatórios periciais, realizados em sede de Direito Civil, por acidente de viação $(\mathrm{n}=156)$.

2. O DS foi considerado apenas em $71 \%$ dos casos em que havia referência a perturbações a nível da sexualidade $(\mathrm{n}=111)$;

2.1. Estas perturbações estavam directamente relacionadas com a função sexual em cerca de $46 \%$ dos casos, admitindo-se a existência de outras alterações (por exemplo, a nível ortopédico), como condicionantes do desempenho sexual e, portanto, justificando a atribuição de DS;

2.2. Em 3 destes casos o DS apenas foi valorado qualitativamente; 
2.3. Em $20 \%$ não foram descritas alterações nem a nível das queixas funcionais, nem situacionais;

2.4. Apenas em 6 casos foram solicitados exames de especialidade para a confirmação deste dano;

2.5. Em nenhum caso foram requeridos, directamente pelo perito, exames complementares de diagnóstico;

2.6. A atribuição de DS não foi justificada no capítulo da "Discussão" em 24\% dos casos;

2.7. Nos casos em que foi apresentada fundamentação, pelo menos em $50 \%$ esta era vaga e, por vezes, desadequada;

2.8. A justificação mais frequente relacionou-se com queixas álgicas não genitais $(25 \%)$.

Tendo em conta estas conclusões, propõe-se o seguinte, relativamente à avaliação e valoração do DS em sede de Direito Civil:

1. Este dano deve ser sempre pesquisado, independentemente da idade, sexo ou estado civil da vítima;

2. Nesse sentido, a vítima deve ser abordada de forma adequada, com delicadeza, mas com profissionalismo, face à possibilidade da sua existência, salvo em casos muito específicos em que o perito considere ser do interesse desta não o fazer. Ainda assim, tal opção deverá constar do relatório;

3. Se necessário e adequado, deve ser também entrevistado(a) sobre o assunto o(a) parceiro(a) sexual da vítima;

4. A descrição e valoração deste dano, a nível do relatório, poderá merecer o consentimento da pessoa a avaliar, se capaz de o dar;

5. A valoração deste dano deve apoiar-se na sua descrição a nível das queixas e, nos casos em que tal for possível, ser objectivado através do exame físico; se essa objectivação carecer da realização de exames de especialidade ou complementares de diagnóstico, estes devem ser efectuados;

6. Se os exames de especialidade considerarem a possibilidade terapêutica das perturbações sexuais, dever-se-á ponderar a questão da data de consolidação;

7. Esta valoração deverá privilegiar a descrição, tanto mais que a Tabela portuguesa de indemnizações não considera este dano; no entanto, atendendo à metodologia preconizada para os restantes danos permanentes, não económicos, propõe-se que seja feita, sempre que possível, a sua quantificação numa escala de 7 graus de gravidade crescente; 
8. Nos casos em que as perturbações sexuais tenham uma evidente componente orgânica e funcional, este dano deverá ser avaliado em duas vertentes, a IPG (de acordo com a Tabela de Incapacidades em Direito Civil) e o DS, tal como acontece actualmente com o Dano Estético;

9. A valoração do DS deve ser sempre justificada no sub-capítulo de danos permanentes, a nível do capítulo da "Discussão", tendo em conta, sempre que possível, as classificações existentes, designadamente o DSM-IV-TR;

10. Deverá ser descrita, na história do evento, relativamente à vivência pós-traumática, a eventual existência de dano sexual temporário;

11. Deverão ser descritos os casos de DS potencial, tendo em vista permitir a reabertura do processo caso este se venha a manifestar.

\section{Bibliografia}

1. Allen Gomes F. Paixão Amor e Sexo. Lisboa: Publicações D. Quixote; 2004. 257 p.

2. American Psychiatry Association. Manual de Diagnóstico e Estatística das Perturbações Mentais. DSM-IV. 4 a ed, Texto Revisto. Lisboa: Climepsi Editores; 2006. 943 p.

3. Foucault M. História da Sexualidade I, A Vontade de Saber. Lisboa: Relógio D’ Água Editores; 1994. 161p.

4. Hatzimouratidis K, and Hatzichristou D. Sexual dysfunctions: Classifications and definitions, J Sex Med 2007; 4: 241-250.

5. Lowy M, Collins S, Bloch M, et al. Quality of Erection Questionnaire correlates. Change in erection quality with erectile function, hardness, and psychosocial measures in men treated with sildenafil for erectile dysfunction. J Sex Med 2007; 4: 83-92.

6. Lucas $\mathrm{P}$, Bargagna $\mathrm{M}$, Boróbia $\mathrm{C}$, et col. La rationalisation de l'évaluation européenne des atteints à la personne humaine. Revista Portuguesa do Dano Corporal 2001; 10(11): 21-35.

7. Magalhães T, Carneiro de Sousa MJ, Matos E, et col. Análise de 1082 casos de acidentes de viação não mortais. In: Vieira DN, Rebelo A, Corte-Real F eds. Coimbra: Temas de Medicina Legal. Centro de estudos de pós-graduação em Medicina Legal; 1998. p. 381-390.

8. Magalhães T, Corte-Real F, Vieira DN. O Relatório Pericial de Avaliação do Dano Pericial em Direito Civil. In: Vieira DN e Quintero JA eds, Aspectos práticos da avaliação do dano corporal em Direito Civil. Coimbra: Caixa Seguros e Imprensa da Universidade de Coimbra; 2008. p. 159-171.

9. Magalhães T, Taveira-Gomes A. Salvar o corpo / Salvar a vida - A morbilidade póstraumática. Arquivos de Medicina 2001; 15(4-5-6): 95-98.

10. Magalhães T. Avaliação do dano corporal em Andrologia. In: Andrologia clínica. Porto: Sociedade Portuguesa de Andrologia; 2000. p. 677-689.

11. Nicolosi A, Buvat J, Glasser DB, et al. For the GSSAB Investigators' Group Sexual behaviour, sexual dysfunctions and related help seeking patterns in middle-aged and 
elderly Europeans: the global study of sexual attitudes and behaviours. World J Urol 2006; 24: 423-428.

12. Sadock BJ, Sadock VA. Kaplan \& Sadock's synopsis of psychiatry: behavioural sciences/ clinical psychiatry. 10th ed. Philadelphia: Lippincott Williams \& Wilkins; 2007. 1470 p.

13. Sidi H, Ezat S, Puteh W, et al. The Prevalence of Sexual Dysfunction and Potential Risk Factors That May Impair Sexual Function in Malaysian Women, International Society for Sexual Medicine. J Sex Med 2005; 4(2):311-321.

14. Vallejo Ruiloba J. Introducción a la Psicopatología y la Psiquiatria. $6^{\mathrm{a}}$ ed. Barcelona: Masson; 2006. 937 p.

15. Vieira DN. O perito e a Missão Pericial em Direito Civil. In: Vieira DN e Quintero JA eds. Aspectos práticos da avaliação do dano corporal em Direito Civil. Coimbra: Caixa Seguros e Imprensa da Universidade de Coimbra; 2008. p. 35-59.

Resumo: 0 dano sexual. Contributo para a avaliação médico-legal dos danos na pessoa $\mathrm{Na}$ prática clínica, deparamo-nos muitas vezes com questões relativas a perturbações do desempenho sexual consequentes a eventos traumáticos. A avaliação deste Dano Sexual (DS) pode ser feita em qualquer âmbito do Direito mas, no caso do Direito Civil, está prevista a sua valoração enquanto parâmetro específico de dano. No entanto, esta avaliação é muito complexa, entre outros motivos, pela sua difícil objectivação e, portanto, pela dificuldade que daí resulta no estabelecimento do nexo de causalidade com o traumatismo. Importa, por isso, que essa avaliação assente numa correcta metodologia e em critérios clínicos rigorosos e bem definidos. 0 presente estudo tem como objectivo analisar os critérios de avaliação e valoração médico-legal do DS, bem como a sua repercussão na decisão judicial. Para tal, realizou-se um estudo retrospectivo, a partir dos relatórios periciais elaborados nos serviços médico-legais do norte de Portugal (Delegação e respectivos Gabinetes Médico-Legais) ( $n=156)$ e uma análise das respectivas sentenças judiciais $(n=47)$. Consideraram-se os seguintes critérios de inclusão: exames no âmbito de Direito Civil, consequentes a acidente de viação, com relatórios únicos e concluídos ou finais, em que havia referência à existência de queixas do foro da sexualidade e/ou valoração do DS. Os resultados revelaram que os casos onde, de alguma forma, é feita referência a perturbações da sexualidade, correspondem a $4 \%$ dos exames efectuados em sede de Direito Civil. Dos 156 casos em que havia referência a uma qualquer perturbação do foro da sexualidade, o DS não foi considerado em 29\% dos casos. Dos 111 casos em que foi considerada a existência de DS, este não foi referenciado no capítulo das "Queixas" em $20 \%$ dos casos ( $n=22$ ) e não foi justificado em $24 \%(n=27)$. Nos casos em que a atribuição do DS foi justificada ( $n=84$ ), essa justificação nem sempre encontrou suporte a nível da sua descrição no capítulo das "Queixas" (19\%). Relativamente às decisões judiciais ( $n=47)$, o DS foi considerado como um parâmetro de dano relevante para a definição da indemnização em apenas 1 caso e, em apenas 9 houve referências a queixas relacionadas com o foro sexual. 0 carácter íntimo da matéria em avaliação, a dificuldade de objectivação do dano e, por vezes, a ausência de abordagem destas situações, conduzem a uma subavaliação do DS. No entanto, este dano pode ter grande relevância para a qualidade de vida dos doentes, pelo que não pode 
deixar de ser diagnosticado, tratado e reparado. As dificuldades inerentes à sua avaliação, demonstradas no presente estudo, são reveladoras da necessidade de um maior trabalho de definição de normas procedimentais quanto aos seus critérios de avaliação, bem como uma melhor formação e sensibilização dos peritos quanto à importância desta matéria.

Palavras-chave: Disfunção sexual; dano sexual; perícia médico-legal.

Summary: Sexual Damage. An approach to the medico-legal assessment of bodily damage In clinical practice, we are frequently faced with sexual performance disturbances following traumatic events. This "Sexual Damage" (SD) assessment can be made by any field of the Law, however, in the case of Common Law, it should be valued as a specific parameter of damage. Nevertheless, this damage assessment is complex, among other reasons because of its objectification difficulties and, therefore, the increased difficulty in the settlement of the causality nexus with the traumatism. Thus, it is important to base that evaluation on a correct methodology and on well-defined and rigorous clinical criteria. The present study aims to analyze forensic SD criteria of evaluation and valorization, as well as its repercussions on the judicial decision. For such, a retrospective study was undertaken, based on expert reports elaborated in the medical-legal services in Northern Portugal (Delegations and Offices) $(n=156)$ and in the respective judicial sentences analysis $(n=47)$. The following inclusion criteria have been considered: Common Law assessment, following a road traffic accident, with "unique" or "finished" reports, in which there was mention of complaints concerning sexuality and/or valorization of SD. The results showed that the cases where, in some way, reference to sexual disturbances was made, amount for $4 \%$ of the examinations conducted in the realm of Common Law. Of the 156 cases in which there was reference to any sexual disturbance, SD was not considered in $29 \%$ of them. Of the 111 cases in which the existence of SD was considered, this was not mentioned in the "Complaints" chapter in 20\% of the cases $(n=22)$ and was not justified in $24 \%(n=27)$. In the cases in which the assessment of SD was justified $(n=84)$, the given justification did not always find support with its description in the "Complaints" chapter (19\%). Concerning judicial decisions $(n=47)$, SD was considered as a relevant damage parameter for the definition of the compensation in just 1 case and, in only 9 cases there were references to complaints related to sexuality. The private nature of the subject being evaluated, the damage objectification difficulties and, at times, the absence of a direct approach to these situations, lead to an under-evaluation of SD. However, this sort of damage can be of great relevance to a patients' quality of life and thus, cannot be undiagnosed, untreated and unrepaired. The difficulties inherent to its evaluation, demonstrated in the present study, reveal the need for a greater effort in defining procedural norms concerning its evaluation criteria, as well as better formation and awareness of experts as to the importance of this matter.

Key-words: Sexual dysfunction; sexual damage; forensic expertise.

Résumé: Dommage Sexuel. Une contribution pour l'évaluation du dommage à la personne Dans la pratique clinique, nous rencontrons souvent des troubles de la performance sexuelle après des événements traumatiques. L'évaluation de ce Dommage Sexuel (DS) peut se faire en tous les domaines du droit, mais il est prévu, en tant que chef de préjudice, dans le 
cadre du droit civil. Toutefois, l'évaluation de ce dommage est vraiment complexe, parmi d'autres raisons, parce que son objectivation est difficile et, donc, ça rend aussi difficile l'établissement de la causalité avec un traumatisme. Il faut, par conséquent, que cette appréciation soit fondée sur une méthodologie saine et sur des critères cliniques rigoureux et bien définis.

La présente étude vise à examiner les critères d'évaluation et de valorisation médico-légale du DS, ainsi que son impact sur la décision judiciaire. On a mené une étude rétrospective, fondée sur des rapports d'experts en services médico-légaux du Nord du Portugal (Délégation et bureaux) ( $n=156)$ et une analyse de leurs jugements ( $n=47)$. On a examiné les critères d'inclusion suivants: les examens dans le cadre du droit civil (conséquences d'accident de circulation), avec des rapports uniques et complets ou définitifs, dans lesquels il était faite une référence à l'existence de plaintes dans la juridiction de la sexualité et / ou de valorisation du DS. Les résultats ont révélé que les cas où il était fait référence, en quelque sorte, à des troubles de la sexualité, correspondent à $4 \%$ des examens réalisés dans le cadre du droit civil. Sur les 156 cas où l'on faisait référence à une perturbation de la sexualité, le DS n'a pas été observé dans $29 \%$ des cas. Sur les 111 cas où l'on a considéré l'existence de DS, celui-ci n'était pas mentionné dans le chapitre concernant les «plaintes» dans 20\% des cas $(n=22)$ et n'était pas justifié dans $24 \%(n=27)$. Dans les cas où l'attribution de DS était justifiée $(n=84)$, celle-ci n'a pas toujours trouvé un appui à sa description dans le chapitre concernant les «plaintes» (19\%). En ce qui concerne les décisions judiciaires $(n=47)$, le DS a été considéré comme un paramètre de dommages pertinents pour la définition de l'indemnisation, seulement dans 1 cas, et seulement dans 9 on a trouvé des références sur les plaintes relatives au forum sexuel. La nature intime de la matière en évaluation, la difficulté d'objectivation des dommages et, parfois, l'absence d'abordage de ces situations conduisent à une sous-estimation du DS. Toutefois, ce type de dommage se revêt d'une grande importance pour la qualité de vie des patients et on ne peut pas éviter qu'il ne soit pas diagnostiqué, traité et réparé. Les difficultés de l'évaluation de ces dommages, démontrées par cette étude, révèlent le besoin de poursuivre les travaux pour une meilleure définition des normes procédurales, en ce qui concerne à leurs critères d'évaluation, ainsi qu'une meilleure formation et sensibilisation des experts concernant l'importance de cette question.

Mots-clés: Dysfonctionnement sexuel; dommage sexuel; expertise médicale.

\section{Pedido de separatas:}

LILIANA SANTOS

lilianagsantosster@gmail.com 